\title{
SYNTHESIS AND ANTICONVULSANT ACTIVITY OF 6-IODO PHTHALAZINEDIONE DERIVATIVES
}

\author{
BY \\ Rezk R. Ayyad ${ }^{(1)}$ and Ahmed M. Monsour ${ }^{(2)}$ \\ FROM \\ ${ }^{(1)}$ Department of Pharmaceutical Chemistry, ${ }^{(2)}$ Department of Pharmacology, Faculty of \\ Pharmacy (Boys), Al-Azhar University, Cairo, Egypt.
}

\section{ABSTRACT.}

A series of phthalazinedione bearing substituted sulfonamide moiety acetanilide esters hydrazide and oxadiazole derivatives $\left(\mathbf{3}_{\mathrm{a}-\mathrm{f}}\right),\left(\mathbf{4}_{\mathrm{a}-\mathrm{g}}\right),\left(\mathbf{5}_{\mathrm{a}-\mathrm{d}}\right),(\mathbf{6}),(\mathbf{7}),(\mathbf{8}),\left(\mathbf{9}_{\mathrm{a}-\mathrm{d}}\right)$ was synthesized in good yield and evaluated for their possible anticonvulsant activity. The structures of the synthesized compounds were confirmed on the basis of their spectral data and elemental analysis. Their anticonvulsant activity was evaluated by the maximal electroshock induced seizure (MES) and subcutaneous pentylenetetrazole (PTZ) tests. All the tested compounds showed considerable anticonvulsant activity in at least one of the tested compounds exhibited moderate anticonvulsant activity in both MES and PTZ tests. From these results, phthalazinedione bearing chloroacetyl benzene sulfonamide $\left(\mathbf{3}_{\mathbf{a}-\mathrm{f}-}\right)$, acetanilide $\left(\mathbf{4}_{\mathrm{a}-\mathrm{g}}\right)$ ester $\left(\mathbf{5}_{\mathrm{a}-\mathrm{d}}\right)$, hydrazide (6), oxadiazole (7) and ester $\left(\mathbf{9}_{\mathrm{a}-\mathrm{d}}\right)$ derivatives could be recommended as novel structures of broad spectrum anticonvulsants.

Key words: Epileptics Anticonvulsant, Iodophthalazindione and Oxadiazole.

\section{Introduction}

Epilepsy is one of the most common neurological disorders, affecting about $1 \%$ of the world's population according to WHO . Many efforts devoted in the recent years for the development of novel therapeutics resulted in the availability of several newer drugs as promising anticonvulsants [1,2]. However, the currently available anticonvulsants are effective in reducing the severity and number of seizures in less than $70 \%$ of patients. Moreover, their usage is associated with undesirable numerous side effects [2-5]. Therefore, continued search for safer and more effective anticonvulsants is urgently necessary.

It is well documented that one of the important core fragments of anticonvulsants is defined by nitrogen heterocyclic system, usually lactam or imide, with attached phenyl or alkyl groups [6]. This common template is found in the structures of first generation anticonvulsant such as ethosuximide, phenytoin, quinazoline and phthalazine derivatives, phthalazines have received much attention in recent years owing to their both biological significance and pharmaceutical applications [7]. Many phthalazine derivatives have been reported as anticonvulsant agents [8]. 5 membered heterocylic compounds show various types of biological activities among them 
substituted sulfonamide moiety wide spectrum of activities such as antibacterial,[9] antimalarial,[10] and anticonvulsant [11-13].

In this study, we describe the synthesis of target compounds as hybrid molecules contain phthalazinedione as potential hydrophobic aryl rings containing nitrogen heterocyclic system required for anticonvulsant activity plus inclusion of substituted oxadiazole functionality to form group of compounds resembling and collecting both features of nitrogen moiety and 5 membered heterocyclic derivatives, in addition, iodine moiety, which has received considerable attention in organic synthesis because of its high tolerance to air and moisture, low-cost nontoxic nature and ready availability, was introduced in the phenyl ring of phthalazine structure to increase the lipophilicity of the molecules, enhance the absorption distribution and increase the activity of target compounds in hope of developing potent and safe new effective anticonvulsant compounds $[14,15]$.

A literature survey revealed that the presence of phthalazine moiety, which undergo substitution at the heteroatom or the distal aromatic ring, is necessary requirement for the CNS depression and anticonvulsant activities of compounds such as compound (1)[1,3] (2)[13,17]. Containing pharmacophoric group-NH-CO which present in quinazoline and quinoxaline reciprocally inarrangement.

On the other hand some substituted derivatives (1) [1,3], and (2) $[13,17]$ were reported to display anticonvulsant activity substitution at position 2 and 5 from the ring. Introduction of a nucleophile at position 2 and a hydrophobic substituent at position 5 considerably increased the anticonvulsant activity [11-13]. In view of the previous rationale, it was thought worthwhile to study the effects of 2 pharmacphoric moieties like phthalazindione and substituted derivatives in a single molecule, on the biological activity. The target compounds have been designed to contain different hydrophobic substituents attached to substituted moiety starting from $\mathrm{H}$ in an ascending order, with acetanilides, sulfonamides esters hydrazide and oxadiazoles linkage to study the effect of hydrophobic change on the biological activity of these compounds as anticonvulsant agents. 
<smiles>CCCC(C)(O)C(O)CC</smiles>

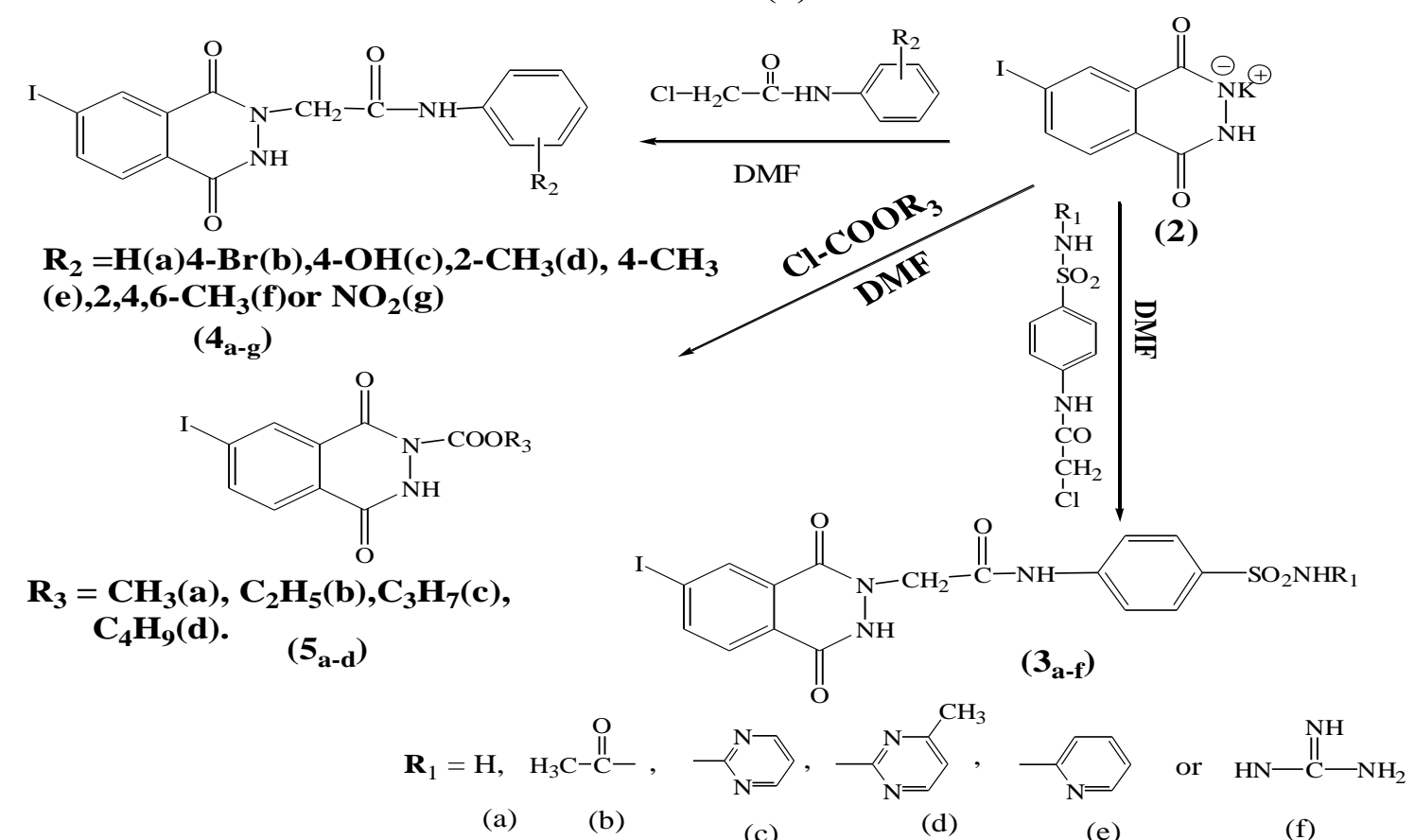<smiles>CCOC(=O)n1[nH]c(=O)c2ccc(I)cc2c1=O</smiles>

$\left(5_{b}\right)$<smiles>NNC(=O)n1[nH]c(=O)c2ccc(I)cc2c1=O</smiles>

(6)

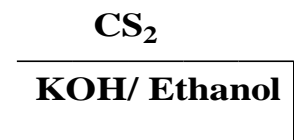
KOH/ Ethanol (6)<smiles>CC(C)(C)CC(=O)O</smiles><smiles>O=c1[nH]n(-c2nnc(S)o2)c(=O)c2cc(I)ccc12</smiles>

(7)

\section{Fig. 1. Target compounds}

Scheme 1 


\section{Materials and methods}

Materials

All melting points measured on a Griffin melting point apparatus (Griffin, Valdosta, GA, USA) and are uncorrected. The infrared spectra were recorded as $\mathrm{KBr}$ discs on a Nicolet IR 200 (Thermo Fisher Scientific, Barrington USA) at the Pharmaceutical Analytical Unit., Faculty of Pharmacy, Al-Azhar University, Cairo Egypt. The ${ }^{1} \mathrm{H}$ NMR spectra were run using TMS as an internal standard (Aldrich chemical Co., Milwaukee, WI, USA) on Varian Mercury VXr-300 NMR (Varian, Palo Alto, CA, USA) at the Micro analytical Center, Faculty of Science, Cairo University, Giza, Egypt. Mass spectra were performed on Varian MAT 311-A (70eV) (Varian, San Fernando, CA, USA) at the Micro analytical Center, Faculty of Science, Cairo University, Giza, Egypt. Elemental analyses $(\mathrm{C}, \mathrm{H}, \mathrm{N})$ were performed on a perkin-Elmer 2400 analyzer (Perkin-Elmer, Norwalk, CT, USA) at the micronalytical unit of Cairo University Giza, Egypt. All compounds were within $\pm 0.5 \%$ of the theoretical values. All chemicals used for synthesis were purchased from (Sigma-Aldrich Chemical Co. Milwaukee, WI, USA).

\section{Synthesis}

The synthetic strategy to prepare the target compounds(1-9) is depicted in Fig.2: 4iodophthalic anhydride was refluxed with hydrazine hydrate in ethanol for $4 \mathrm{~h}$ to afford quantitative yield of 2,3-dihydro-6-iodophthalazine-1,4-dione (1), which was refluxed with alcoholic potassium hydroxide in ethanol to furnish potassium salt of 2,3-dihydro-6iodophthalazine-1,4-dione (1) in 85\% yield. Compound (2) was reacted with substituted chloroacetyl benzene sulfonamide,substituted acetanilides and alkylchloroformate in anhydrous DMF to give compounds $\left(\mathbf{3}_{\mathrm{a}-\mathrm{f}}\right),\left(\mathbf{4}_{\mathrm{a}-\mathrm{g}}\right),\left(\mathbf{5}_{\mathrm{a}-\mathrm{d}}\right)$. Compound $\left(\mathbf{5}_{\mathrm{b}}\right)$ treated with hydrazine hydrate afford (6) which condensed with $\mathrm{CS}_{2}$ and $\mathrm{KOH}$ to give (7). The compound (7) striated with alcoholic $\mathrm{KOH}$ resulted (8) which react with different alkyl chloroacetate to give $\left(\mathbf{9}_{\text {a-d }}\right)$ (Scheme 1).

\section{2,3-Dihydro-6-iodophthalazine-1,4-dione(1) $[1,3]$.}

It was prepared by refluxing $(2,74 \mathrm{~g}, 0.01 \mathrm{~mol})$ of 4-iodophthalic anhydride with hydrazine hydrate $(0.49 \mathrm{ml}$. $)$ in ethanol $(50 \mathrm{ml}$. $)$ for $4 \mathrm{hs}$, left to cool, collected, filtered and crystallized from ethanol. Yield 90\%, mp.; 290-2 ${ }^{\circ} \mathrm{C} ;{ }^{1} \mathrm{H}$ NMR $\left(\mathrm{CDCl}_{3}, 300 \mathrm{MHz}\right) \delta: 7.23-8.16$ $(\mathrm{m}, 5 \mathrm{H}, \mathrm{Ar}-\mathrm{H}, 2 \mathrm{NH}), \mathrm{C}_{8} \mathrm{H}_{5} \mathrm{IH}_{2} \mathrm{O}_{2}: \mathrm{Ms}(70 \mathrm{eV}): \mathrm{m} / \mathrm{z}=89(\mathrm{M}+1)$.

\section{Potassium salt of 2,3-dihydro-6-iodophthalazine-1,4-dione (2) [13,16].}

$(2.89 \mathrm{~g}-0.01 \mathrm{~mol})$ of compound (1) was dissolved in ethanol $(50 \mathrm{ml}$.) and $\mathrm{KOH}(0.56 \mathrm{~g}-$ $0.01 \mathrm{~mol})$ was dissolved in ethanol $(10 \mathrm{ml})$, the latter solution was added to the former to give the potassium salt of 2,3-dhydro-6-iodophthalazine-1,4-dione (2) which crystallized from ethanol. Yield 85\%, mp: $290-2 \mathrm{C}^{\circ}$; ${ }^{1} \mathrm{H}$ NMR $\left(\mathrm{D}_{2} \mathrm{O}, 300 \mathrm{MHz}\right) \delta: 7.02-7.94(\mathrm{~m}, 3 \mathrm{H}, \mathrm{Ar}-\mathrm{H})$, $\mathrm{C}_{8} \mathrm{H}_{4} \mathrm{IKN}_{2} \mathrm{O}_{2}$ : $\mathrm{MS}(70 \mathrm{eV}): \mathrm{m} / \mathrm{z}=327(\mathrm{M}+1)$.

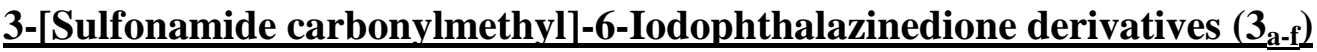

In a conical flask $(0.01 \mathrm{~mol}, 3.2 \mathrm{~g})$ of compound (2) mixed with the appropriate amount $(0.01 \mathrm{~mol})$ of different chloroacetyl benzene sulfonamides in dimethylformamide (DMF) (100 $\mathrm{ml}$ ), refluxed for $2 \mathrm{hs}$, cooled then poured on crushed ice. The crystals collected by filtration, recrystallized from ethanol m.p. yield, elemental analysis table I 
Table 1

\begin{tabular}{|c|c|c|c|c|c|c|c|}
\hline \multirow{2}{*}{$\begin{array}{l}\text { Comp.N } \\
\text { o . }\end{array}$} & \multirow{2}{*}{$\mathbf{R}$} & \multirow{2}{*}{$\begin{array}{l}\text { M.P. } \\
{ }^{\circ} \mathrm{C}\end{array}$} & \multirow{2}{*}{$\begin{array}{l}\text { Yield } \\
(\mathrm{gm}) \%\end{array}$} & \multirow{2}{*}{$\begin{array}{l}\text { Molecular formula } \\
\text { M. Wt }\end{array}$} & \multicolumn{3}{|c|}{ Elemental analyses } \\
\hline & & & & & $\%$ & Calcd. & Found \\
\hline $\mathbf{3}_{\mathrm{a}}$ & $\mathrm{H}$ & $\begin{array}{l}242- \\
243\end{array}$ & $83 \%(4.15)$ & $\begin{array}{l}\mathrm{C}_{16} \mathrm{H}_{13} \mathrm{IN}_{4} \mathrm{O}_{5} \mathrm{~S} \\
500\end{array}$ & $\begin{array}{l}\mathrm{C} \% \\
\mathrm{H} \% \\
\mathrm{~N} \%\end{array}$ & $\begin{array}{l}38.30 \\
2.60 \\
11.10\end{array}$ & $\begin{array}{l}38.19 \\
2.45 \\
11.05\end{array}$ \\
\hline $\mathbf{3}_{\mathrm{b}}$ & $\stackrel{\mathrm{O}}{-\mathrm{C}-\mathrm{CH}_{3}}$ & $\begin{array}{l}262- \\
264\end{array}$ & $77 \%(4.17)$ & $\begin{array}{l}\mathrm{C}_{18} \mathrm{H}_{15} \mathrm{IN}_{4} \mathrm{O}_{6} \mathrm{~S} \\
542\end{array}$ & $\begin{array}{l}\mathrm{C} \% \\
\mathrm{H} \% \\
\mathrm{~N} \%\end{array}$ & $\begin{array}{l}39.85 \\
2.77 \\
10.33\end{array}$ & $\begin{array}{l}40.06 \\
2.55 \\
10.45\end{array}$ \\
\hline $\mathbf{3}_{\mathrm{c}}$ & & $\begin{array}{l}233- \\
234\end{array}$ & $\begin{array}{l}82 \% \\
(4.75) \\
\end{array}$ & $\begin{array}{l}\mathrm{C}_{20} \mathrm{H}_{15} \mathrm{IN}_{6} \mathrm{O}_{5} \mathrm{~S} \\
578\end{array}$ & $\begin{array}{l}\mathrm{C} \% \\
\mathrm{H} \% \\
\mathrm{~N} \%\end{array}$ & $\begin{array}{l}41.52 \\
2.60 \\
14.53 \\
\end{array}$ & $\begin{array}{l}41.14 \\
2.76 \\
14.87 \\
\end{array}$ \\
\hline $3_{d}$ & & $\begin{array}{l}269- \\
271\end{array}$ & $\begin{array}{l}55 \% \\
(3.25) \\
\end{array}$ & $\begin{array}{l}\mathrm{C}_{21} \mathrm{H}_{17} \mathrm{IN}_{6} \mathrm{O}_{5} \mathrm{~S} \\
592\end{array}$ & $\begin{array}{l}\mathrm{C} \% \\
\mathrm{H} \% \\
\mathrm{~N} \% \\
\end{array}$ & $\begin{array}{l}42.57 \\
2.87 \\
14.19 \\
\end{array}$ & $\begin{array}{l}42.25 \\
3.32 \\
14.05 \\
\end{array}$ \\
\hline $\mathbf{3}_{3}$ & & $\begin{array}{l}251- \\
252\end{array}$ & $\begin{array}{l}73 \% \\
(4.21) \\
\end{array}$ & $\begin{array}{l}\mathrm{C}_{21} \mathrm{H}_{16} \mathrm{IN}_{5} \mathrm{O}_{5} \mathrm{~S} \\
577\end{array}$ & $\begin{array}{l}\mathrm{C} \% \\
\mathrm{H} \% \\
\mathrm{~N} \% \\
\end{array}$ & $\begin{array}{l}43.67 \\
2.77 \\
12.13 \\
\end{array}$ & $\begin{array}{l}44.80 \\
2.98 \\
12.11 \\
\end{array}$ \\
\hline $3_{\mathrm{f}}$ & $\begin{array}{c}-\mathrm{NH}-\mathrm{C}-\mathrm{NH}_{2} \\
\mathrm{NH}\end{array}$ & $\begin{array}{l}245- \\
247\end{array}$ & $\begin{array}{l}52 \% \\
(2.9) \\
\end{array}$ & $\begin{array}{l}\mathrm{C}_{17} \mathrm{H}_{16} \mathrm{IN}_{7} \mathrm{O}_{5} \mathrm{~S} \\
557\end{array}$ & $\begin{array}{l}\mathrm{C} \% \\
\mathrm{H} \% \\
\mathrm{~N} \% \\
\end{array}$ & $\begin{array}{l}36.62 \\
2.87 \\
17.59 \\
\end{array}$ & $\begin{array}{l}36.21 \\
3.21 \\
17.83 \\
\end{array}$ \\
\hline
\end{tabular}

2-(N-sulfanilamid carbonylmethyl)-6-iodophthalazine-1,4-dione $\left(3_{\mathrm{a}}\right)$

${ }^{1} \mathrm{HNMR} \delta\left(\mathrm{D}_{2} \mathrm{O}\right) 4.8\left(1 \mathrm{H}, \mathrm{CH}_{2}-\mathrm{COO}-\right) 7.2-8(\mathrm{~m}, 7 \mathrm{H}$ of aromatic ring $), 10.3(\mathrm{~s}, 1 \mathrm{H} \mathrm{NH}$ of ring), 5.2(s,1H, NH-COO-), 5.1( $\left.\mathrm{s}, \mathrm{SO}_{2} \mathrm{NH}_{2}\right)$.

Ms (70ev)m/z 500 (10\%), 287, $\left.\mathrm{C}_{8} \mathrm{H}_{4} \mathrm{IN}_{2} \mathrm{O}_{2} 100 \%\right)$.

\section{2-(N-sulfacetamido carbonylmethyl)-6-iodophthalazine-1,4-dione ( $3_{\mathrm{b}}$ )}

${ }^{1} \mathrm{HNMR} \delta\left(\mathrm{D}_{2} \mathrm{O}\right) 4.95\left(\mathrm{~s}, 2 \mathrm{H}-\mathrm{CH}_{2}-\mathrm{COO}-\right), 5-5.3(\mathrm{NH}), 10.2(\mathrm{~s}, 1 \mathrm{H}, \mathrm{NH}$ of ring), $1.5(\mathrm{~s}, 3 \mathrm{H}$, $\left.\mathrm{COO}-\mathrm{CH}_{3}\right)$.

Ms (70ev)m/z 542 (15\%), 43-COO-CH$\left.(18 \%), 499, \mathrm{C}_{16} \mathrm{H}_{12} \mathrm{IN}_{2} \mathrm{O}_{2} \mathrm{~S} 20 \%\right)$.

\section{2-(N-sulfadiazino carbonylmethyl)-6-iodophthalazine-1,4-dione (3 $\mathrm{c}$ )}

${ }^{1} \mathrm{HNMR} \delta\left(\mathrm{D}_{2} \mathrm{O}\right) 4.9\left(\mathrm{~s}, 2 \mathrm{H}-\mathrm{CH}_{2}-\mathrm{COO}-\right), 5.2(\mathrm{~s}, 1 \mathrm{H}, \mathrm{NH}), 10.5(\mathrm{~s}, 1 \mathrm{H}, \mathrm{NH}$ of ring), 7.6-8.1 $(\mathrm{m}, 10 \mathrm{H}$ of aromatic).

Ms (70ev)m/z 500(M+1) (10\%), 287, $\mathrm{C}_{8} \mathrm{H}_{4} \mathrm{IN}_{2} \mathrm{O}_{2}(100 \%), 291 \mathrm{C}_{12} \mathrm{H}_{11} \mathrm{~N}_{4} \mathrm{O}_{3} \mathrm{~S}(17 \%)$.

\section{2-(N-sulfamethazino)-6-iodophthalazine-1,4-dione ( $\left.\mathbf{3}_{\mathrm{d}}\right)$}

${ }^{1} \mathrm{HNMR} \delta\left(\mathrm{D}_{2} \mathrm{O}\right) 4.95\left(\mathrm{~s}, 2 \mathrm{H}-\mathrm{CH}_{2}-\mathrm{COO}-\right), 1.3\left(\mathrm{~s}, 3 \mathrm{H}-\mathrm{CH}_{3}\right), 5.1(\mathrm{~s}, 1 \mathrm{H}-\mathrm{NH}), 10.5(\mathrm{~s}, 1 \mathrm{H}, \mathrm{NH}$ of ring $) 7.5-8.2(\mathrm{~m}, 2 \mathrm{H}$ aromatic protons $)$.

Ms (ev)m/z 594(M+2) (23\%), 287, $\mathrm{C}_{8} \mathrm{H}_{4} \mathrm{IN}_{2} \mathrm{O}_{2}(100 \%), 305 \mathrm{C}_{13} \mathrm{H}_{13} \mathrm{~N}_{4} \mathrm{O}_{3} \mathrm{~S}(9 \%)$.

\section{2-(N-sulfapyridino)-6-iodophthalazine-1,4-dione ( $\mathbf{3}_{\mathrm{e}}$ )}

${ }^{1} \mathrm{HNMR} \delta\left(\mathrm{D}_{2} \mathrm{O}\right) 4.88\left(\mathrm{~s}, 2 \mathrm{H}-\mathrm{CH}_{2}-\right), 1.5(\mathrm{~s}, 1 \mathrm{H}-\mathrm{NH}), 10.5(\mathrm{~s}, 1 \mathrm{H}-\mathrm{NH}$ of ring $), 7.6-8.2(\mathrm{~m}, 2 \mathrm{H}$ aromatic protons).

Ms (ev)m/z 577 (33\%), 287, $\mathrm{C}_{8} \mathrm{H}_{4} \mathrm{IN}_{2} \mathrm{O}_{2}$ (100\%), $290 \mathrm{C}_{13} \mathrm{H}_{12} \mathrm{~N}_{5} \mathrm{O}_{3} \mathrm{~S}(7 \%)$.

\section{2-(N-sulfaguanidino)-6-iodophthalazine-1,4-dione $\left(\mathbf{3}_{\mathrm{f}}\right)$}

${ }^{1} \mathrm{HNMR} \delta\left(\mathrm{D}_{2} \mathrm{O}\right) 4.9\left(\mathrm{~s}, 2 \mathrm{H}-\mathrm{CH}_{2}-\right), 7.5-8.2(\mathrm{~m}, 7 \mathrm{H}$ aromatic protons $), 10.5(\mathrm{~s}, 1 \mathrm{H}, \mathrm{NH}$ of ring), 5.2(s, $1 \mathrm{H}, \mathrm{NH}), 4.2\left(\mathrm{~s}, 2 \mathrm{H}-\mathrm{NH}_{2}\right)$.

Ms (ev)m/z 557(M) (13\%), 287, $\mathrm{C}_{8} \mathrm{H}_{4} \mathrm{IN}_{2} \mathrm{O}_{2}(100 \%), 270 \mathrm{C}_{9} \mathrm{H}_{12} \mathrm{~N}_{5} \mathrm{O}_{3} \mathrm{~S}(17 \%)$. 


\section{3-Anilinocarbonylmethyl-6-iodophthalazinedione (4 $\left.\mathbf{a}_{\mathrm{a}-\mathrm{g}}\right)$.}

In aconical flask (0.01 mol, 3.2gm) of compound (2) mixed with the appropriate amount of acetanilide derivatives $(0.01 \mathrm{~mol})$ in $100 \mathrm{ml}$ of diemthylfomamide (DMF), refluxed for $3 \mathrm{hrs}$ then cooled, poured on crushed ice. The crystals collected by filtration, re-crystallized from ethanol yield, m.p. elemental analyses in table 2

Table 2

\begin{tabular}{|c|c|c|c|c|c|c|c|}
\hline \multirow{2}{*}{$\begin{array}{l}\text { Comp.N } \\
\text { o . }\end{array}$} & \multirow{2}{*}{$\mathbf{R}$} & \multirow{2}{*}{$\begin{array}{l}\text { M.P. } \\
{ }^{\circ} \mathrm{C}\end{array}$} & \multirow{2}{*}{$\begin{array}{l}\text { Yield } \\
(\text { gm) } \%\end{array}$} & \multirow{2}{*}{$\begin{array}{l}\text { Molecular formula } \\
\text { M. Wt }\end{array}$} & \multicolumn{3}{|c|}{ Elemental analyses } \\
\hline & & & & & $\%$ & Calcd. & Found \\
\hline $4 a_{a}$ & $\mathrm{H}$ & $\begin{array}{l}272- \\
274\end{array}$ & $92 \%(3.87)$ & $\begin{array}{l}\mathrm{C}_{16} \mathrm{H}_{12} \mathrm{IN}_{3} \mathrm{O}_{3} \\
421\end{array}$ & $\begin{array}{l}\mathrm{C} \% \\
\mathrm{H} \%\end{array}$ & $\begin{array}{l}45.61 \\
2.85\end{array}$ & $\begin{array}{l}45.23 \\
3.13 \\
\end{array}$ \\
\hline $4_{b}$ & $4-\mathrm{Br}$ & $\begin{array}{l}229- \\
231\end{array}$ & $\begin{array}{l}87 \% \\
(4.35)\end{array}$ & $\begin{array}{l}\mathrm{C}_{16} \mathrm{H}_{11} \mathrm{BrIN}_{3} \mathrm{O}_{3} \\
500\end{array}$ & $\begin{array}{l}\mathrm{C} \% \\
\mathrm{H} \%\end{array}$ & $\begin{array}{l}38.40 \\
2.20\end{array}$ & $\begin{array}{l}37.95 \\
2.55\end{array}$ \\
\hline $4_{c}$ & $4-\mathrm{OH}$ & $\begin{array}{l}235- \\
236\end{array}$ & $\begin{array}{l}73 \% \\
(3.19)\end{array}$ & $\begin{array}{l}\mathrm{C}_{16} \mathrm{H}_{12} \mathrm{IN}_{3} \mathrm{O}_{4} \\
437\end{array}$ & $\begin{array}{l}\mathrm{C} \% \\
\mathrm{H} \%\end{array}$ & $\begin{array}{l}43.94 \\
2.75\end{array}$ & $\begin{array}{l}43.71 \\
3.11\end{array}$ \\
\hline $4_{d}$ & $2-\mathrm{CH}_{3}$ & $\begin{array}{l}289- \\
291\end{array}$ & $\begin{array}{l}77 \% \\
(3.73)\end{array}$ & $\begin{array}{l}\mathrm{C}_{17} \mathrm{H}_{14} \mathrm{IN}_{3} \mathrm{O}_{3} \\
435\end{array}$ & $\begin{array}{l}\mathrm{C} \% \\
\mathrm{H} \%\end{array}$ & $\begin{array}{l}46.89 \\
3.22\end{array}$ & $\begin{array}{l}46.91 \\
2.75\end{array}$ \\
\hline $4_{e}$ & $4-\mathrm{CH} 3$ & $\begin{array}{l}301- \\
302\end{array}$ & $\begin{array}{l}81 \% \\
(3.52)\end{array}$ & $\begin{array}{l}\mathrm{C}_{17} \mathrm{H}_{14} \mathrm{IN}_{3} \mathrm{O}_{3} \\
435\end{array}$ & $\begin{array}{l}\mathrm{C} \% \\
\mathrm{H} \%\end{array}$ & $\begin{array}{l}46.89 \\
3.22 \\
\end{array}$ & $\begin{array}{l}47.12 \\
3.38 \\
\end{array}$ \\
\hline $\mathbf{4}_{\mathrm{f}}$ & $\begin{array}{l}\text { 2,4,3-Tri- } \\
\text { methyl }\end{array}$ & $294-$ & $\begin{array}{l}89 \% \\
(4.12)\end{array}$ & $\begin{array}{l}\mathrm{C}_{19} \mathrm{H}_{18} \mathrm{IN}_{3} \mathrm{O}_{3} \\
463\end{array}$ & $\begin{array}{l}\mathrm{C} \% \\
\mathrm{H} \%\end{array}$ & $\begin{array}{l}49.24 \\
3.89\end{array}$ & $\begin{array}{l}49.33 \\
4.21\end{array}$ \\
\hline $4_{g}$ & $4-\mathrm{NO}_{2}$ & $\begin{array}{l}241- \\
243\end{array}$ & $\begin{array}{l}71 \% \\
(3.31)\end{array}$ & $\begin{array}{l}\mathrm{C}_{16} \mathrm{H}_{11} \mathrm{IN}_{4} \mathrm{O}_{5} \\
466\end{array}$ & $\begin{array}{l}\mathrm{C} \% \\
\mathrm{H} \%\end{array}$ & $\begin{array}{l}41.20 \\
2.36\end{array}$ & $\begin{array}{l}40.89 \\
1.93\end{array}$ \\
\hline
\end{tabular}

\section{2-(Anilinocarbonylmethyl)-6-iodophthalazinedion $\left(4_{\mathrm{a}}\right)$}

$\left(\mathrm{CDCl}_{3} 300 \mathrm{MHz}\right) \quad \delta \quad 4.01\left(\mathrm{~d}, \quad 2 \mathrm{H}, \mathrm{CH}_{2}\right), \quad\left(\mathrm{N}-\mathrm{CO}-\mathrm{CH}_{2}\right), 7.50-8.4(\mathrm{~m}, \quad 8 \mathrm{H}$ aromatic protons), 10.51 (s, $2 \mathrm{H}-\mathrm{NH}$ of the ring and amide group).

IR $\mathrm{cm}^{-1} 3173(\mathrm{NH}), 1725$ (carbonyl group of amide and 1664-1580 carbonyl of ring. Ms (70ev): $\mathrm{m} / \mathrm{z}$ 422(M+1)(65\%), 287 (421-( $\left.\mathrm{C}_{8} \mathrm{H}_{8} \mathrm{NO}\right)(134(33 \%)$.

\section{2-(4-Bromoanilinocarbonylmethylphthalainedione) $\left(4_{b}\right)$}

${ }^{1} \mathrm{HNMR} \quad\left(\mathrm{CDCl}_{3} \mathrm{MHz}\right) \quad \delta \quad 4.8\left(\mathrm{~d}, 2 \mathrm{H}, \quad \mathrm{CH}_{2}-\mathrm{CO}-\right), \quad 7.6-8.4(\mathrm{~m}, 7 \mathrm{H}$ aromatic proton), (s,1H,10.3, NH of ring).

IR $\mathrm{cm}^{-1} 3099 \mathrm{NH}, 1681-\mathrm{CO}-\mathrm{N}$ and 1660-1590-CO of ring) Ms (70ev)m/z 499(M-1) (32\%) 501(M+) (63\%), $287 \mathrm{C}_{8} \mathrm{H}_{4} \mathrm{IN}_{2} \mathrm{O}_{2}(100 \%)$.

\section{2-(4-Hydroxyanilino-6-iodophthalazinedion) $\left(4_{c}\right)$}

${ }^{1} \mathrm{HNMR}\left(\mathrm{CDCl}_{3} \mathrm{MHz}\right) \delta 9.25(\mathrm{~s}, 1 \mathrm{H}$, of $\mathrm{OH}), 4.95\left(\mathrm{~d}, 2 \mathrm{H}, \mathrm{CH}_{2}-\mathrm{CO}\right), 7.8-8.2(7 \mathrm{H}$ of aromatic ring), (s,1H,10.3, $\mathrm{NH}$ of ring).

IR $\mathrm{cm}^{-1} 3050 \mathrm{NH}, 1680-\mathrm{NH}-\mathrm{CO}, 1655-1628 \mathrm{CO}$ of ring, Ms (70ev)m/z 436(M-1) (20\%) $287(100 \%)$.

\section{2-(2-Methylanilinocarbonylmethyl)-6-iodophthalazinedion $\left(4_{d}\right)$}

${ }^{1} \mathrm{HNMR}\left(\mathrm{CDCl}_{3} 300 \mathrm{MHz}\right) \delta 2.2\left(\mathrm{~s}, 3 \mathrm{H}, 2-\mathrm{CH}_{3}\right), 4.8\left(\mathrm{~s}, 2 \mathrm{H},-\mathrm{CH}_{2^{-}}\right), 7.1-8.4(\mathrm{~m}, 7 \mathrm{H}$, aromatic protons), $10.8(\mathrm{~s}, 1 \mathrm{H}, \mathrm{NH}$ of ring), 5.2 ( $\mathrm{s}, 1 \mathrm{H}$ of amide).

IR cm ${ }^{-1} 3182(\mathrm{NH}), 1698(\mathrm{CO}-\mathrm{NH}-)$ 1650-1590(-CO of ring) Ms (70ev)m/z 435, 148, $\mathrm{C}_{9} \mathrm{H}_{10} \mathrm{NO}(41 \%), 287 \mathrm{C}_{8} \mathrm{H}_{4} \mathrm{IN}_{2} \mathrm{O}_{2}(100 \%)$.

\section{2-(4-Methylanilinocarbonylmethyl)-6-iodophthalazinedione $\left(4_{\mathrm{e}}\right)$}

${ }^{1} \mathrm{HNMR}\left(\mathrm{CDCl}_{3} 300 \mathrm{MHz}\right) \delta 2.15\left(\mathrm{~s}, 3 \mathrm{H}, 4-\mathrm{CH}_{3}\right), 4.9\left(\mathrm{~s}, 2 \mathrm{H},-\mathrm{CH}_{2}-\right), 7.2-8.3(\mathrm{~m}, 7 \mathrm{H}$, aromatic protons), (s, $10.4 \mathrm{NH}$ of ring).

IR cm cm $^{-1}$ 3177(NH), 1690(CO-NH-) 1655-1600(-CO of ring) Ms (70ev)m/z 436(M+1). 


\section{2-(2,4,6-Trimetylanilinocarbonylmethyl)-6-iodophthalazinedione $\left(4_{\mathrm{f}}\right)$}

${ }^{1} \mathrm{HNMR}\left(\mathrm{CDCl}_{3} 300 \mathrm{MHz}\right) 4.95\left(\mathrm{~s}, \mathrm{NH}, \mathrm{CO}\right.$, side chaing), $1.9\left(\mathrm{~s}, 3 \mathrm{H},-\mathrm{CH}_{3^{-}}\right), 7.8-8.5(\mathrm{~m}$, $5 \mathrm{H}$, aromatic protons), (s,10.3 $\mathrm{NH}$ of ring)..

Ms m/z (464(M+1), 27\%, $176 \mathrm{C}_{11} \mathrm{H}_{5} \mathrm{NO}(11 \%), 287(100 \%)$.

\section{2-(4-Nitroanilinocarbonylmethyl)-6-iodophthalazinedione $\left(4_{\mathrm{g}}\right)$}

${ }^{1} \mathrm{HNMR}\left(\mathrm{CDCl}_{3} 300 \mathrm{MHz}\right) 4.75\left(\mathrm{~s}, 1 \mathrm{H}, \underline{\mathrm{NH}}-\mathrm{CH}_{2}-\mathrm{CO}\right), 7.7-8.4(\mathrm{~m}, 7 \mathrm{H}$, aromatic ring), 10.5 (s, $1 \mathrm{H}-\mathrm{NH}$ of ring)..

Ms (70ev)m/z 465(M-1), 19\%, 287(100\%), $179 \mathrm{C}_{8} \mathrm{H}_{7} \mathrm{~N}_{2} \mathrm{O}_{3}(15 \%)$.

\section{3-Akoxycarbonyl-6-iodophthalazine $(2 \mathrm{H}, 3 \mathrm{H})-1,4-d i o n e ~\left(5_{\mathbf{a}-\mathrm{d}}\right)$}

In a conical flask $(0.01 \mathrm{~mol}, 3.2 \mathrm{gm})$ of compound (2) mixed with the appropriate amount of alkyl (methyl, ethyl, propyl or butyl)chloro formate in (DMF) $100 \mathrm{ml}$ refluxed for $2 \mathrm{hrs}$, cooled then pouredon crushed ice. The crystals collected by filtration recrystallized from ethanol. The yield, m.p. and elemental analysis at table 3.

Table 3.

\begin{tabular}{|c|c|c|c|c|c|c|c|}
\hline \multirow{2}{*}{$\begin{array}{l}\text { Comp.N } \\
\text { o. }\end{array}$} & \multirow{2}{*}{$\mathbf{R}$} & \multirow{2}{*}{$\begin{array}{l}\text { M.P. } \\
{ }^{0} \mathrm{C}\end{array}$} & \multirow{2}{*}{$\begin{array}{l}\text { Yield } \\
(\mathrm{gm}) \%\end{array}$} & \multirow{2}{*}{$\begin{array}{l}\text { Molecular formula } \\
\text { M. Wt }\end{array}$} & \multicolumn{3}{|c|}{ Elemental analyses } \\
\hline & & & & & $\%$ & Calcd. & Found \\
\hline $5 \mathrm{a}$ & $-\mathrm{CH}_{3}$ & $190-2$ & $\begin{array}{l}80 \% \\
(2.82)\end{array}$ & $\begin{array}{l}\mathrm{C}_{10} \mathrm{H}_{7} \mathrm{~N}_{2} \mathrm{IO}_{4} \\
346\end{array}$ & $\begin{array}{l}\mathrm{C} \% \\
\mathrm{H} \% \\
\mathrm{~N} \%\end{array}$ & $\begin{array}{l}34.68 \\
2.02 \\
8.09\end{array}$ & $\begin{array}{l}34.87 \\
1.72 \\
8.02\end{array}$ \\
\hline $5_{b}$ & $-\mathrm{C}_{2} \mathrm{H}_{5}$ & $160-1$ & $\begin{array}{l}95 \% \\
(3.49) \\
\end{array}$ & $\begin{array}{l}\mathrm{C}_{11} \mathrm{H}_{9} \mathrm{~N}_{2} \mathrm{IO}_{4} \\
360\end{array}$ & $\begin{array}{l}\mathrm{C} \% \\
\mathrm{H} \% \\
\mathrm{~N} \% \\
\end{array}$ & $\begin{array}{l}36.67 \\
2.50 \\
7.78 \\
\end{array}$ & $\begin{array}{l}36.17 \\
2.18 \\
8.12 \\
\end{array}$ \\
\hline $5_{c}$ & $-\mathrm{C}_{3} \mathrm{H}_{7}$ & $183-1$ & $\begin{array}{l}75 \% \\
(3.62) \\
\end{array}$ & $\begin{array}{l}\mathrm{C}_{12} \mathrm{H}_{11} \mathrm{~N}_{2} \mathrm{IO}_{4} \\
374\end{array}$ & $\begin{array}{l}\mathrm{C} \% \\
\mathrm{H} \% \\
\mathrm{~N} \%\end{array}$ & $\begin{array}{l}38.50 \\
2.94 \\
7.49\end{array}$ & $\begin{array}{l}38.41 \\
3.05 \\
7.31\end{array}$ \\
\hline $5_{d}$ & $-\mathrm{C}_{4} \mathrm{H}_{9}$ & $150-2$ & $\begin{array}{l}80 \% \\
(3.16)\end{array}$ & $\begin{array}{l}\mathrm{C}_{13} \mathrm{H}_{13} \mathrm{~N}_{2} \mathrm{IO}_{4} \\
388\end{array}$ & $\begin{array}{l}\mathrm{C} \% \\
\mathrm{H} \% \\
\mathrm{~N} \%\end{array}$ & $\begin{array}{l}40.20 \\
3.35 \\
7.22 \\
\end{array}$ & $\begin{array}{l}40.18 \\
3.19 \\
6.88 \\
\end{array}$ \\
\hline
\end{tabular}

$\mathbf{5}_{\mathrm{a}}:{ }^{1} \mathrm{HNMR}\left(\mathrm{CDCl}_{3} 300 \mathrm{MHz}\right) \delta 1.4\left(\mathrm{t}, \mathrm{J}=8,3 \mathrm{H}, \mathrm{CH}_{3}\right), 6.98-7.7(\mathrm{~m}, 3 \mathrm{H}, \mathrm{Ar}-\mathrm{H}) .8 .28(\mathrm{~s}, 1 \mathrm{H}, \mathrm{NH})$, $\mathrm{Ms}(70 \mathrm{ev}) \mathrm{m} / \mathrm{z} 353(\mathrm{M}+) 354 \mathrm{~m} / \mathrm{z}\left(-\mathrm{OCH}_{3}\right) 325$.

$\mathbf{5}_{\mathbf{b}}:{ }^{1} \mathrm{HNMR}\left(\mathrm{CDCl}_{3} 300 \mathrm{MHz}\right) \delta 1.5\left(\mathrm{t}, \mathrm{J}=8.1,3 \mathrm{H}, \mathrm{CH}_{3}\right), 4.4\left(\mathrm{q}, \mathrm{J}=7.6, \mathrm{~J}=2.52 \mathrm{H} \mathrm{CH}_{2},\right), 7.2-7.8$ $(\mathrm{m}, 3 \mathrm{H}, \mathrm{Ar}-\mathrm{H}) .8 .23(\mathrm{~s}, 1 \mathrm{H}, \mathrm{NH})$,

Ms (70ev)m/z 367(M-1) 366.

5: ${ }^{1} \mathrm{HNMR}\left(\mathrm{CDCl}_{3} 300 \mathrm{MHz}\right) \delta 1.3\left(\mathrm{t}, \mathrm{J}=8.2,3 \mathrm{H}, \mathrm{CH}_{3}\right), 4.4\left(\mathrm{q}, \mathrm{J}=7.6, \mathrm{~J}=2.5,2 \mathrm{H}, \mathrm{CH}_{2}\right), 2.5$ (q, $\left.\mathrm{J}=2.1, \mathrm{~J}=8.22 \mathrm{H}, \mathrm{CH}_{2}-\mathrm{CH}_{2}\right), 7.4-8.1(\mathrm{~m}, 3 \mathrm{H}, \mathrm{Ar}-\mathrm{H}) .8 .15(\mathrm{~s}, 1 \mathrm{H}, \mathrm{NH})$,

Ms (70ev)m/z 381(M+1, 382).

5 $_{\mathrm{d}}:{ }^{1} \mathrm{HNMR}\left(\mathrm{CDCl}_{3} 300 \mathrm{MHz}\right) \delta 1.1\left(\mathrm{t}, \mathrm{J}=7.8,3 \mathrm{H}, \mathrm{CH}_{3}\right), 1.3-1.6(\mathrm{~m}, 3 \mathrm{H}, \mathrm{Ar}-\mathrm{H}) .1 .7-1.9(\mathrm{~m}, 2 \mathrm{H}$, $\left.\mathrm{CH}_{2}\right), 2.55\left(\mathrm{t}, \mathrm{J}=82 \mathrm{H}, \mathrm{CH}_{2}\right), 4.1\left(\mathrm{~s}, 2 \mathrm{H}, \mathrm{CH}_{2}\right), 4.55\left(\mathrm{~s}, 2 \mathrm{H}, \mathrm{CH}_{2}\right), 7.1-7.9(\mathrm{~m}, 3 \mathrm{H}, \mathrm{Ar}-\mathrm{H})$, $8.3(\mathrm{~s}, \mathrm{NH}, 1 \mathrm{H})$

Ms (70ev)m/z 394(M+2, 398).

\section{3-(Hydroazinocarbonyl)-6-Iodophthalazine( $2 \mathrm{H}, 3 \mathrm{H})$-1,4-dione (6).}

A mixture of ethanolic solution $(50 \mathrm{gm})$ of $5 \mathrm{~b}(0.01 \mathrm{~mol}, 3.7 \mathrm{gm})$ and of hydrazine hydrate $(1 \mathrm{ml})$ heated with stirring at $100^{\circ} \mathrm{C}$ for $30 \mathrm{~min}$. then the mixture filtered, washed with ethanol the yield $88 \%$, m.p. $245-7^{\circ} \mathrm{C}$

${ }^{1} \mathrm{HNMR}\left(\mathrm{D}_{2} \mathrm{O}, 300 \mathrm{MHz}\right) \quad \delta \quad 4.47\left(\mathrm{~s}, 2 \mathrm{H}, \mathrm{CH}_{2}\right), 5.2(\mathrm{~s}, 1 \mathrm{H}, \mathrm{NH}), 4.9\left(\mathrm{~s}, 2 \mathrm{H}, \mathrm{NH}_{2}\right), \quad 7.02-$ 8.1(m,3H,Ar-H),

Ms (70ev)m/z 353(M+1) 354(M-N $\left.\mathrm{H}_{2}\right) 322$. 
3-(Potassium salt of 2-mercapto oxadiazole-5-yl-6-iodophthalazine-(2H,3H)-1,4-dione and 2-Mercaptoxadiazole-5-yl-iodophthalazine (2H,3H) -1,4-dione (7) and (8).

A mixture of absolute (100\%) (100 ml) ethanolic solution of compound VI (0.02 mol, $7.14 \mathrm{gm})(0.02 \mathrm{~mol}, 1.44 \mathrm{~m}) \mathrm{l}$ of carbon disulfide and (0.02 mol, $1.12 \mathrm{gm})$ of $\mathrm{KOH}$ refluxed for $6 \mathrm{hrs}$, cooled, poured half solution on acidified water $\left(\mathrm{H}_{2} \mathrm{O}, \mathrm{HCl} 1: 2\right)$ and the other half of solution filtered as it is the two resulted 2-mercapto and 2-potassium salt respectively.

2-Mercapto oxadiazole-5-yl-6-iodophthalazine-(2H,3H)-1,4-dione (7), yield 90\% m.p. 303-4, Molecular formula $\mathrm{C}_{10} \mathrm{H}_{5} \mathrm{IN}_{4} \mathrm{O}_{3} \mathrm{~S}$, mol weight 388, calc, $\mathrm{C}=3.92, \mathrm{H}=1.24, \mathrm{~N}=14.43$ and found $\mathrm{C}=3.42, \mathrm{H}=0.96, \mathrm{~N}=13.91$.

Compound (7): ${ }^{1} \mathrm{HNMR}\left(\mathrm{CDCl}_{3} 300 \mathrm{MHz}\right) 7.4-8.1(\mathrm{~m}, 3 \mathrm{H}, \mathrm{Ar}-\mathrm{H}), 8.2(\mathrm{~s}, 1 \mathrm{H}, \mathrm{NH}), 3.2(\mathrm{~s}, 1 \mathrm{H}, \mathrm{SH})$ $\mathrm{C}_{10} \mathrm{H}_{5} \mathrm{IN}_{4} \mathrm{O}_{3} \mathrm{~S}:$ Ms $(70 \mathrm{ev}) \mathrm{m} / \mathrm{z} 388(\mathrm{M}+1,398)$.

Compound (8) potassium salt of 3-(2-mercaptooxadiazole-5-yl)-6-iodophthalazine $(2 \mathrm{H}, 3 \mathrm{H})-1,4-$ dione, yield 82\%, m.p. $>330$ mol. Formula $\mathrm{C}_{10} \mathrm{H}_{4} \mathrm{IKN}_{4} \mathrm{O}_{3} \mathrm{~S}$, mol. Wt. 426.

\section{3-[(2-Alkoxycarbonylthio)oxadiazole-5-yl]-6-iodophthalazine $(2 \mathrm{H}, 3 \mathrm{H})-1,4$-dione $(9$ a-d $)$.}

In aconical flask compound (8) $(0.01 \mathrm{~mol}, 4.3 \mathrm{gm})$ mixed with the appropriate amount of alkylchloroformate derivatives $(0.01 \mathrm{~mol})$ in (DMF) $100 \mathrm{ml}$ refluxed for $2 \mathrm{hrs}$ then cooled, poured on crushed ice, the crystals collected by filtration re-crystallized from ethanol the m.p. yield elemental analysis at table 4

Table 4

\begin{tabular}{|c|c|c|c|c|c|c|c|}
\hline \multirow{2}{*}{$\begin{array}{l}\text { Comp.N } \\
\text { o. }\end{array}$} & \multirow{2}{*}{$\mathbf{R}$} & \multirow{2}{*}{$\begin{array}{l}\text { M.P. } \\
{ }^{0} \mathrm{C}\end{array}$} & \multirow{2}{*}{$\begin{array}{l}\text { Yield } \\
(\mathrm{gm}) \%\end{array}$} & \multirow{2}{*}{$\begin{array}{l}\text { Molecular formula } \\
\text { M. Wt }\end{array}$} & \multicolumn{3}{|c|}{ Elemental analyses } \\
\hline & & & & & $\%$ & Calcd. & Found \\
\hline $9 \mathrm{a}$ & $\mathrm{CH}_{3}$ & $160-2$ & $\begin{array}{l}75 \% \\
(3.45)\end{array}$ & $\begin{array}{l}\mathrm{C}_{13} \mathrm{H}_{9} \mathrm{~N}_{4} \mathrm{IO}_{5} \mathrm{~S} \\
460\end{array}$ & $\begin{array}{l}\mathrm{C} \% \\
\mathrm{H} \% \\
\mathrm{~N} \%\end{array}$ & $\begin{array}{l}33.91 \\
1.96 \\
12.17 \\
\end{array}$ & $\begin{array}{l}34.01 \\
2.04 \\
11.88\end{array}$ \\
\hline $9_{b}$ & $-\mathrm{C}_{2} \mathrm{H}_{5}$ & $141-3$ & $\begin{array}{l}90 \% \\
(4.27)\end{array}$ & $\begin{array}{l}\mathrm{C}_{14} \mathrm{H}_{11} \mathrm{~N}_{4} \mathrm{IO}_{5} \mathrm{~S} \\
474\end{array}$ & $\begin{array}{l}\mathrm{C} \% \\
\mathrm{H} \% \\
\mathrm{~N} \%\end{array}$ & $\begin{array}{l}35.44 \\
2.32 \\
11.81\end{array}$ & \begin{tabular}{|l|}
35.39 \\
1.96 \\
11.40
\end{tabular} \\
\hline $9 \mathrm{c}$ & $-\mathrm{C}_{3} \mathrm{H}_{7}$ & $172-4$ & $\begin{array}{l}70 \% \\
(3.42)\end{array}$ & $\begin{array}{l}\mathrm{C}_{15} \mathrm{H}_{13} \mathrm{~N}_{4} \mathrm{IO}_{5} \mathrm{~S} \\
488\end{array}$ & $\begin{array}{l}\mathrm{C} \% \\
\mathrm{H} \% \\
\mathrm{~N} \%\end{array}$ & $\begin{array}{l}36.88 \\
2.66 \\
11.48\end{array}$ & $\begin{array}{l}37.31 \\
2.66 \\
11.40\end{array}$ \\
\hline $9_{d}$ & $-\mathrm{C}_{4} \mathrm{H}_{9}$ & $187-8$ & $\begin{array}{l}65 \% \\
(3.26)\end{array}$ & $\begin{array}{l}\mathrm{C}_{16} \mathrm{H}_{15} \mathrm{~N}_{4} \mathrm{IO}_{5} \mathrm{~S} \\
502\end{array}$ & $\begin{array}{l}\mathrm{C} \% \\
\mathrm{H} \% \\
\mathrm{~N} \%\end{array}$ & $\begin{array}{l}38.24 \\
2.99 \\
11.15\end{array}$ & $\begin{array}{l}38.10 \\
3.05 \\
10.91\end{array}$ \\
\hline
\end{tabular}

9: $\quad{ }^{1} \mathrm{HNMR}\left(\mathrm{CDCl}_{3} 300 \mathrm{MHz}\right) \delta 1.5\left(\mathrm{~s}, 3 \mathrm{H}, \mathrm{CH}_{3}\right), 7.6-8.1(\mathrm{~m}, 3 \mathrm{H}, \mathrm{Ar}-\mathrm{H}), 8.3(\mathrm{~s}, 1 \mathrm{H}, \mathrm{NH})$, Ms (70ev) $\mathrm{m} / \mathrm{z}$ 460(M+1) 461

9 $: \quad{ }^{1} \mathrm{HNMR}\left(\mathrm{CDCl}_{3} 300 \mathrm{MHz}\right) \delta 1.2\left(\mathrm{t}, \mathrm{J}=7.8,3 \mathrm{H}, \mathrm{CH}_{3}\right), 2.5\left(\mathrm{q}, \mathrm{J}=2.2, \mathrm{~J}=8.2-2 \mathrm{H}, \mathrm{CH}_{2}\right), 7.3-$ 8.2(m,3H, Ar-H), 8.2(s, 1H, NH).

Ms (70ev)m/z 474(M+2), 476, M- $\mathrm{C}_{2} \mathrm{H}_{5}(445)$

9: $\quad{ }^{1} \mathrm{HNMR}\left(\mathrm{CDCl}_{3} 300 \mathrm{MHz}\right) \delta 1.1\left(\mathrm{t}, \mathrm{J}=7.8,3 \mathrm{H}, \mathrm{CH}_{3}\right), 1.7-1.9\left(\mathrm{~m}, 2 \mathrm{H}, \mathrm{CH}_{2}\right), 2.6(\mathrm{t}, \mathrm{J}=8,2 \mathrm{H}$, $\left.\mathrm{CH}_{2}\right)$, 7.1-7.6 (m,3H, Ar-H), 8.4(s, 1H , NH).

Ms (70ev)m/z 488(M+1), 489.

9d: $\quad{ }^{1} \mathrm{HNMR}\left(\mathrm{CDCl}_{3} 300 \mathrm{MHz}\right) \delta 1.1\left(\mathrm{t}, \mathrm{J}=7.91,3 \mathrm{H}, \mathrm{CH}_{3}\right), 1.4-1.7\left(\mathrm{~m}, 2 \mathrm{H}, \mathrm{CH}_{2}\right), 7.2-7.7(\mathrm{~m}, 3 \mathrm{H}-$ Ar-H), 8.3(s, 1H, NH).

Ms (70ev)m/z 474(M+1), 305. 


\section{Anticonvulsant activity}

The animal studies were undertaken with approval from Al-Azhar University, Cairo, Egypt. Ethics committee (approval \# 29PD/3/9/4M. all the trials were carried out according to the respective internationally valid guidelines. The anticonvulsant activities are usually evaluated by both the MES and PTZ tests [18]. The MES test is related to the generalized tonicclonic seizure and PTZ test to the absence seizure [2,18]. Thus, these two kinds of anticonvulsant tests are meaningful for the clinical prediction of the anticonvulsant drug candidates and evaluation of their anticonvulsant spectrum $[3,18]$. Therefore, the anticonvulsant evaluation for the tested compounds was carried out by both the MES and PTZ tests according to the protocol of the antiepileptic drug development program. National Institute of Neurological Disorder and Stroke[18]. The reference drugs were used according to the reported method[19].

Adult male white Swiss albino mice weighing 20-25g(10-12 weeks old) were obtained from experimental Animal Care Centre, College of Pharmacy, Al-Azhar University. The animals were maintained under standard conditions of humidity, temperature $(25 \pm 2 \mathrm{C})$ and light (12h light/12hs. dark). They wee fed with a standard mice pellet diet and had free access to water. Each treatment group and vehicle control group consisted of 4 animals. All tested compounds were dissolved in polyethylene glycol 400 and administered intraperitoneally (ip) to ICR male mice at dose of $25,50,75$ and $100 \mathrm{mg} / \mathrm{kg} 30 \mathrm{~min}$ before seizures induction. Diphenylhydantion $(200 \mathrm{mg} / \mathrm{kg})$, methosuximide $(200 \mathrm{mg} / \mathrm{kg})$, sodium valproate $(300 \mathrm{mg} / \mathrm{kg})$ and trimethadione $(00 \mathrm{mg} / \mathrm{kg})$ were used as reference drugs. The seizure was artificially induced by either pentylenetetazole or electric shock. The pentylenetetazole induced seizure (PTZ) test entailed the subcutaneous administration of $80 \mathrm{mg} / \mathrm{kg}$ of pentyleneterazole as a $0.5 \%$ solution in the posterior midline of the mice, and the animal was observed for 60 min. protection was defined as the failure to observe even at threshold seizure (single episode of clonic spasms of at least $5 \mathrm{~s}$ duration). The maximal electroshock seizure (MES) test was elicited with a 60-cycle a.c. of $50 \mathrm{~mA}$ intensity delivered for $0.2 \mathrm{~s}$ via corneal electrodes with ECT unit (UGO Baseline, Italy). A drop of $0.9 \%$ saline was instilled in the eye prior to application of electrodes. Protection in this test was defined as the abolition of the hind limb tonic extension component of the seizure. The percentage protection per each dose and the dose makes protection for $50 \%$ of animals $\left(\mathrm{ED}_{50}\right)$ were calculated using INSTAT 2 program (ICS, Philadelphia, PA, USA). Observation time with all convulsants was 60 min for convulsions and death. The animals that showed no convulsion within $1 \mathrm{~h}$ after convulsive drug administration were considered to be protected.

\section{RESULTS}

The results of anticonvulsant activities for tested compounds are summarized in the table 2: From the biological results of $\left(\mathrm{ED}_{50} \mathrm{mmol} / \mathrm{kg}\right)$ listed in the table 2, we notice that increasing the size of alkyl group from $\mathrm{H}$ to methyl leads to increasing the activity and then goes down by further increasing to the alkyl side chain. As seen also in table 5 acetanilides (sulfa drugs, anilins) derivatives, $\left(\mathbf{5}_{\mathbf{b}}\right),(\mathbf{6})$, (7) and $\left(\mathbf{9}_{\mathbf{b}}\right)$ from the synthesized compounds testes as anticonvulsant agents. Against minimal electrical shok MES and pentyleneterazole (PTZ) exhibited considerable anticonvulsant activities in the MES and their $\mathrm{ED}_{50}$ these pharmacological results were comparable to those of anticonvulsant in clinical use but the acetanilides (sulfa drug) showed moderate anticonvulsant and the esters $\left(\mathbf{5}_{\mathbf{b}}\right),\left(\mathbf{9}_{\mathbf{c}}\right)$ hydrazide $(\mathbf{6})$ 2-mercapto (7) and acetanilide $\left(\mathbf{4}_{\mathrm{a}-\mathrm{d}}\right)$ showed aggreable results comparable to the anticonvulsant cilinical use. 
Table 5

\begin{tabular}{|c|c|c|c|c|c|}
\hline Test Comp. & $\mathbf{R}$ & $\begin{array}{l}\text { MES }^{\mathrm{a}} \\
\mathrm{ED}_{50} \% \\
\mathrm{Mg} / \mathrm{kg}\end{array}$ & $\begin{array}{l}\text { MES } \\
\text { ED }_{50} \\
\mathrm{mmol} / \mathrm{kg}\end{array}$ & $\begin{array}{l}\text { PTZ }^{\mathrm{a}} \\
\mathrm{ED}_{50} \% \\
\mathbf{M g} / \mathbf{k g}\end{array}$ & $\begin{array}{l}\text { PTZ } \\
\text { ED }_{50} \% \\
\mathrm{mmol} / \mathrm{kg}\end{array}$ \\
\hline $\mathbf{3}_{\mathrm{a}}$ & $\mathrm{H}$ & 190 & 0.38 & 105 & 0.21 \\
\hline $\mathbf{3}_{\mathrm{b}}$ & $\mathrm{N}_{4}$-acetyl & 197 & 0.36 & 110 & 0.20 \\
\hline $3 \mathbf{3}_{\mathrm{c}}$ & $\mathrm{N}_{4}$-primid-2-yl & 207 & 0.36 & 121 & 0.21 \\
\hline $\mathbf{3}_{\mathrm{d}}$ & $\mathrm{N}_{4}$-3-methazin-2-yl & 202 & 0.34 & 120 & 0.20 \\
\hline $\mathbf{3}_{\mathrm{e}}$ & $\mathrm{N}_{4}$-pyridin-2-yl & 190 & 0.33 & 118 & 0.20 \\
\hline $\mathbf{3}_{\mathrm{f}}$ & $\mathrm{N}_{4}$-gunidin-2-yl & 221 & 0.40 & 130 & 0.23 \\
\hline $\mathbf{4}_{\mathrm{a}}$ & $\mathrm{H}$ & 58 & 0.14 & 30 & 0.07 \\
\hline $\mathbf{4}_{\mathrm{b}}$ & 4-bromo & 55 & 0.12 & 28 & 0.06 \\
\hline $\mathbf{4}_{\mathrm{c}}$ & 4-hydroxy & 62 & 0.14 & 33 & 0.08 \\
\hline $\mathbf{4}_{d}$ & 2-methyl & 69 & 0.16 & 37 & 0.06 \\
\hline 4 & 4-methyl & 75 & 0.17 & 41 & 0.09 \\
\hline $\mathbf{4}_{\mathrm{f}}$ & 2,4,6-trimethyl & 80 & 0.17 & 44 & 0.10 \\
\hline $\mathbf{4}_{\mathrm{g}}$ & 4-nito & 102 & 0.22 & 56 & 0.12 \\
\hline$\overline{5_{b}}$ & ethyl & 30 & 0.110 & 30 & 0.07 \\
\hline 6 & hydrazino & 25 & 0.071 & 28 & 0.06 \\
\hline 7 & mercabto & 40 & 0.10 & 33 & 0.08 \\
\hline \multirow[t]{5}{*}{$\overline{9 c}$} & n-bropyl & 30 & 0.061 & 37 & 0.06 \\
\hline & Diphenylhydantoin & 10.9 & 0.043 & 6.2 & 0.024 \\
\hline & Methosuximide & 45.1 & 0.222 & 35.8 & 0.176 \\
\hline & Sodium Valproate & 283.6 & 1.706 & 154.5 & 0.91 \\
\hline & Trimethadione & 705 & 4.925 & 260.4 & 1.819 \\
\hline
\end{tabular}

\section{Conclusion}

In conclusion, a series of novel structures of Phthalazinedione derivatives were designed, synthesized and their anticonvulsant activities were evaluated by both the MES and PTZ test. This series of compounds was designed as broad spectrum anticonvulsant by hybridizing important moieties such as nitrogen heterocyclic system represented by phthalazine system and substituted oxadiazole group in a single molecule. This was based on the inspection of the structural characteristics in typical anticonvulsants in clinical practice. The $\mathrm{ED}_{50}$ values for these designed compounds were comparable to clinically used antiepileptic drugs. Moreover, some of the designed compounds in this study exhibited considerable anticonvulsant activities in both the MES and PTZ tests. These pharmacological activities were sufficient for them to develop as broad spectrum anticonvulsants. Therefore, it was concluded that, from (4) to (9) derivatives were recommended as novel structures of broad spectrum anticonvulsant.

\section{REFERENCES}

Stefan H., Feuerstein JT.(2007): Novel anticonvulsant drugs. Pharmacol. There 113:165-183.

Donner JF., Snead CO.(2006): New generation anticonvulsants for the treatment of epilepsy in children. NeuroRX; 3: 170-180. 
Loscher W., Sclunidt D.(2002): New horizons in the development of antiepileptic drugs, Epilepsy Res; 50: 3-16.

Loscher W., Sclunidt D.(2006): New horizons in the development of antiepileptic drugs, Innovative strategies. Epilepsy Res; 69:183-272.

Greenwood SR.(2000): adverse effects of antiepileptic drugs. Epilepsia; 41: 42-52.

Wong GM., Defina AJ. Andrews RP.(1986): Conformational analysis of clinically active anticonvulsant drugs. J. Med Chem.; 9: 62-72.

Pelletier CJ, Hesson PD. Jones AK (1996): et.al. substituted 1,2-dihydrophthalazine: Potent, selective and noncompetitive inhibitors of the AMPA receptor; J. Med. Chem.; 39:34-46.

Grasso S., Sarro DG, Sarro DA. (2000): et.al. Synthesis and anticonvulsant activity of novel and potent6,7-methylenedioxyphthalazin-1(2H)-ones, J. Med. Chem.; 43:851-859.

Xu W., HanF., He M (2012): et.al. Inhibition of tobacco bacterial wilt with sulfone derivatives containing an 1,3,4-oxadiazolw Moiety. J. Agric Food Chem.; 60:1036-1041.

Bader H., Hoops FJ. Biel HJ.(1969): et.al. Antimalarial compounds related to diaminodiphenyl sulfone. J. Med. Chem.; 12: 709-711

Watjen F., Baker R., Engelstoff M.(1989): et.al. Novel benzodiazepine receptor partial agonist: oxadiazolylimidazobenodiazepines. J. Med. Chem.; 32: 282-291.

Jhal K, Kumar, Y., Shaharyar M(2010): et.al. Microwave assisted synthesis, characterization and biological evaluation of Benzimidazole substituted 3,4-oxadiazole. Int. J. Chem.Tech Res.; 2:716-727.

Single P., Sharma KP., Sharma KJ.(2012): et. al. Synthesis and evaluation of substituted diphenyl-1,3,4-oxadiazole derivatives for central nervous system depressant activity. Org. Med. Chem.. Lett.; 2(8):1-10.

Laznicek M., Beno P. Waisser K.(1985): et.al. Quantitative chemical structure pharmacokinetic data relationships. IV Relationships between pharmacokinetic data and lipophilicity of iodine-substituted aromatic and arylaliphatic compounds. CeskoSolvenska Farmacie; 34(9): 353-358.

Yamning W., Chester AM. Guo-Feng H.(2003): et. al. Effects of lipophilicity on the affinity and nonspecific binding of iodinated benzothiazole derivatives, J. mol neurosci; 20(3): 255-260. 
Silvakumar R., Gnanasam KS., Ramachandran S(2002): et. al. Pharmacological evaluation of some new 1-substituted-4-hydroxyphthalazine, Eur. J. Med. Chem.; 37: 793-801.

Parsons GC., Danysz, W., Quack NG(1997): et. al. Systemically Active Antagonists of the Glycine Site of the N-methyl-D-aspartate Receptor Electrophysiological, Biochemical and Behavioral Characterization. J. Pharmacol Exp. Ther.; 283:1264-1275.

Krall LR, Penary KJ. White GB(1978): et. al. Antiepileptic drug development H. anticonvulsant drug screening. Epilepsia; 19: 409-428.

Witak TD. Seth K. S., Baizman RE(1972): et. al. p-Substituted N-arcetyl-L-(S)-and D®alpha-amino-N phenlsuccinimides and glutaimides. Substituent effects on stereoselective anticonvulsant activity J. Med. Chem.; 15: 1117-1123.

Rezk Rezk Ayyad Synthesis and Biological Evaluation of Novel iodophthalazinedione derivatives as anticonvulsants Az.J.Pharm. Sci. Vol. 45.

M. Zayed and R.R. Ayyad (2012): some Novel anticonvulsant agents Drived from phthalazinedione (arzneimittelforschung) AMF[2012-04-0067]27.8.: 62: 1-5.

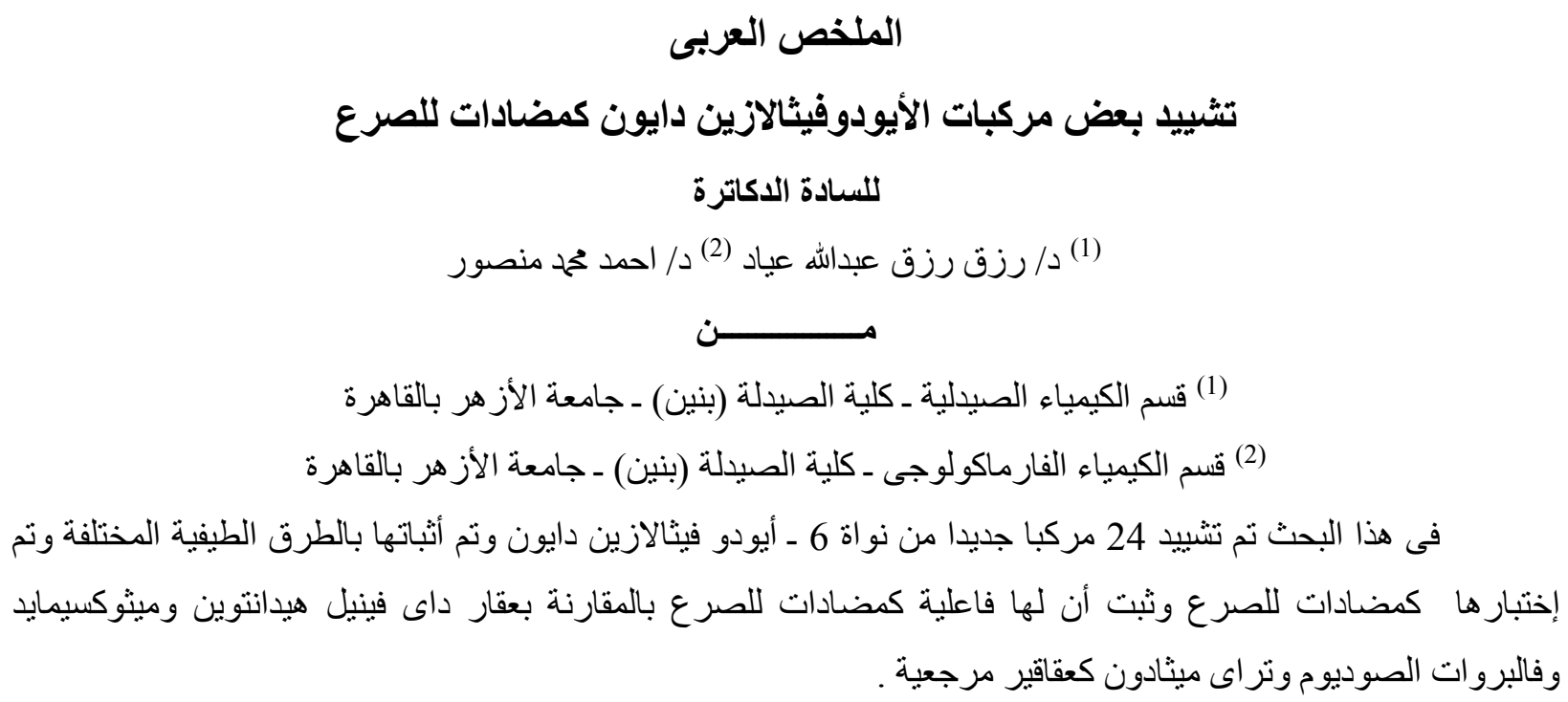

